\title{
Qualitative Analysis of Cosmological Models in $f(R)$-Gravity
}

\author{
R. M. Avagyan, E. V. Chubaryan, G. H. Harutyunyan, \\ A. A. Saharian* \\ Department of Physics, Yerevan State University, 1 Alex Manoogian Street, 0025 Yerevan, \\ Armenia \\ *E-mail: saharian@ysu.am
}

\begin{abstract}
The spatially flat cosmological models are investigated within the framework of $f(R)$-gravity. An equivalent representation in the form of a scalar-tensor theory is discussed. For the general case of the $f(R)$ function, the corresponding cosmological equations are presented in the form of a third order autonomous dynamical system. The qualitative analysis of the latter is given in the absence of nongravitational matter. Various special cases of the function $\mathrm{F}(\mathrm{R})$ are considered and the corresponding phase portraits are depicted. The possibility for the realization of a phase with accelerated expansion of the Universe is discussed.
\end{abstract}

Keywords: $f(R)$-gravity - scalar-tensor theories - cosmological models.

\section{Introduction}

It is known that suitable modifications of General Relativity can result in an accelerating expansion of the Universe at present epoch. These modifications fall into two general groups. The first one consists of scalar-tensor theories that are most widely considered extensions of General Relativity (Will 2018). In addition to the metric tensor, these theories contain scalar fields in their gravitational sector and typically arise in the context of models with extra dimensions (Kaluza-Klein-type models, braneworld scenario) and within the framework of the low-energy string effective gravity. In the second group of models, the Ricci scalar $R$ in the Einstein-Hilbert action is replaced by a general function $f(R)$ (for recent reviews see Nojiri \& Odintso (2007) and Clifton et al. (2012). One of the first models for inflation with quadratic in the Ricci scalar Lagrangian, proposed by Starobinsky (1980), 
falls into this class of theories. An additional motivation for the $f(R)$ theories comes from quantum field theory in classical curved backgrounds (Birrell \& Davies (1982), Buchbinder et al. (1992) and from string theories. The recent investigations of cosmological models in the $f(R)$ theories of gravity have shown a possibility for a unified description of the inflation and the late-time acceleration.

$f(R)$-gravities can be recast as scalar tensor theories of a special type with a potential determined by the form of the function $f(R)$. Various special forms of this function have been discussed in the literature. In particular, the functions were considered that realize the cosmological dynamics with radiation dominated, matter dominated and accelerated epoch. Unified models of inflation and dark energy have been studied as well (Nojiri \& Odintsov 2011). In the present paper we consider the qualitative evolution of the cosmological model for a general $f(R)$ function. The general analysis is specified for various examples, including the original Starobinsky model.

\section{2. $f(R)$-gravity as a scalar-tensor theory}

The action in $(D+1)$-dimensional $f(R)$ theory of gravity has the form

$$
S=\int d^{D+1} x \sqrt{|g|}\left[f(R)+L_{m}\left(g_{i k}, \psi\right)\right]
$$

where $L_{m}\left(g_{i k}, \psi\right)$ is the Lagrangian density for non-gravitational matter collectively denoted by $\psi$. It is well known (see Nojiri \& Odintsov (2011) and Clifton et al. (2012)) that (1) can be presented in the form of the action for scalar-tensor gravity. In order to show that we consider the action

$$
S=\int d^{D+1} x \sqrt{|g|}\left[f^{\prime}(\eta)(R-\eta)+f(\eta)+L_{m}\left(g_{i k}, \psi\right)\right],
$$

with a scalar field $\eta$. The equation for the latter is reduced to $f^{\prime \prime}(\eta)(R-\eta)=$ 0 . Assuming that $f^{\prime \prime}(\eta) \neq 0$, from the field equation we get $\eta=R$. With this solution, the action (2) is reduced to the original action (1).

Introducing a new scalar field $\varphi=-f^{\prime}(\eta)$, the action (2) is written in the form

$$
S=\int d^{D+1} x \sqrt{|g|}\left[-\varphi R-V(\varphi)+L_{m}\left(g_{i k}, \psi\right)\right]
$$

with the scalar potential

$$
V(\varphi)=-f(\eta(\varphi))-\varphi \eta(\varphi) .
$$

Here, we have assumed that the function $\varphi(\eta)$ is invertible. The action (3) describes a scalar-tensor theory. In the representation (3) the Lagrangian density of the non-gravitational matter does not depend on the scalar field $\varphi$. Hence, the representation corresponds to the Jordan frame. 
By the conformal transformation

$$
g_{i k}=\Omega^{2}(\varphi) g_{(E) i k}, \Omega(\varphi)=m_{P} \varphi^{1 /(1-D)},
$$

where $m_{P}=1 /\left(16 \pi G_{D+1}\right)^{1 /(D-1)}$ is the Planck mass in $(D+1)$-dimensions and $G_{D+1}$ is the corresponding gravitational constant, the action is presented as

$$
\begin{aligned}
S= & \int d^{D} x \sqrt{\left|g_{(E)}\right|}\left[-m_{P}^{D-1} R_{(E)}+\frac{1}{2} g_{(E)}^{i k} \partial_{i} \phi \partial_{k} \phi\right. \\
& \left.-V_{E}(\phi)+L_{m}^{E}\left(\phi, g_{(E) i k}, \psi\right)\right] .
\end{aligned}
$$

Here we have introduced a scalar field

$$
\phi=\phi_{0} \ln \left(\varphi / m_{P}^{D-1}\right), \phi_{0}=m_{P}^{(D-1) / 2} \sqrt{2 D /(D-1)},
$$

with a canonical kinetic term and with the potential

$$
V_{E}(\phi)=-\exp \left(-\frac{D+1}{D-1} \frac{\phi}{\phi_{0}}\right)[f(\eta(\varphi))+\varphi \eta(\varphi)], \varphi=m_{P}^{D-1} e^{\phi / \phi_{0}} .
$$

The non-gravitational Lagrangian density is expressed as

$$
L_{m}^{E}\left(\phi, g_{(E) i k}, \psi\right)=\Omega^{D+1} L_{m}\left(\Omega^{2} g_{(E) i k}, \psi\right), \Omega=\exp \left[-\frac{\phi}{(D-1) \phi_{0}}\right] .
$$

In the new conformal frame, referred as the Einstein frame, the gravitational part of the action takes the form of that for $(D+1)$-dimensional General Relativity. In this frame there is a direct interaction between the nongravitational matter and the scalar field. Several examples of the function $f(R)$ with the corresponding potentials will be discussed below.

\section{Cosmological model and the dynamycal system}

Consider a homogeneous and isotropic cosmological model described by the Einstein frame action (6). The corresponding line element has the form

$$
d s_{E}^{2}=d t^{2}-a^{2}(t) d l^{2}
$$

where $d l$ is the line element of a $D$ - dimensional space of constant curvature, $a(t)$ is the scale factor. From the homogeneity of the model it follows that the scalar field should also depend on time only, $\phi=\phi(t)$. The energymomentum tensor corresponding to the metric (10) is diagonal and can be presented in the perfect fluid form $T_{i}^{k}=\operatorname{diag}(\varepsilon, \ldots,-p, \ldots)$, where $\varepsilon$ is the energy density and $p$ is the effective pressure.

Introducing dimensionless quantities $x=\phi / \phi_{0}, \tau=t / t_{0}$, with $t_{0}$ being a positive constant with the dimension of time, for expanding models with 
a flat space the set of cosmological equations is written in terms of the third order autonomous dynamical system

$$
\begin{aligned}
& \frac{d x}{d \tau}=y, \frac{d y}{d \tau}=-b y\left[2 \epsilon+y^{2}+2 V(x)\right]^{1 / 2}+\phi_{0} \alpha \epsilon-V^{\prime}(x) \\
& \frac{d \epsilon}{d \tau}=-\left\{b(1+w)\left[2 \epsilon+y^{2}+2 V(x)\right]^{1 / 2}+\phi_{0} \alpha y\right\} \epsilon
\end{aligned}
$$

where $w=p / \varepsilon, \epsilon=\left(t_{0} / \phi_{0}\right)^{2} \varepsilon, b=D /(D-1)$, and

$$
V(x)=\left(t_{0} / \phi_{0}\right)^{2} V_{E}\left(\phi_{0} x\right), \alpha=\frac{1}{\varepsilon \sqrt{\left|g_{(E)}\right|}} \frac{\delta L_{m}^{E} \sqrt{\left|g_{(E)}\right|}}{\delta \phi} .
$$

The Einstein frame Hubble function $H=a^{-1} d a / d t$ is expressed in terms of the variables of the dynamical system (11) as

$$
H^{2}=\frac{2 \epsilon+y^{2}+2 V(x)}{(D-1)^{2} t_{0}^{2}} .
$$

The set of equations (11) describes the cosmological dynamics in the Einstein frame (for qualitative analysis of cosmological models of string effective gravity in the Einstein frame see Saharian (1999, 2000a). The corresponding dynamics in the Jordan frame is obtained by using the conformal transformation (5). For the line element in the Jordan frame one has $d s_{J}^{2}=d t_{J}^{2}-a_{J}^{2}\left(t_{J}\right) d l^{2}$, where the comoving time coordinate and the scale factor are related to the corresponding Einstein frame quantities by $d t_{J}=m_{P} \varphi^{1 /(1-D)} d t$ and $a_{J}\left(t_{J}\right)=m_{P} \varphi^{1 /(1-D)} a(t)$. For the Hubble function in the Jordan frame we get

$$
H_{J}=\frac{e^{\phi /\left(\phi_{0}(D-1)\right)}}{(D-1) \phi_{0}}\left[ \pm \sqrt{2 \varepsilon+(d \phi / d t)^{2}+2 V_{E}(\phi)}-\frac{d \phi}{d t}\right]
$$

where the upper/lower sign corresponds to expanding/contracting models in the Einstein frame. From the relation (14) it follows that for $V_{E}(\phi)+$ $\varepsilon>0$ the expansion/contraction in the Einstein frame corresponds to the expansion/contraction in the Jordan frame ( $H$ and $H_{J}$ have the same sign).

\section{Qualitative analysis of gravi-scalar models}

The dynamical system (11) has an invariant phase plane $\varepsilon=0$ which corresponds to the pure gravi-scalar models. For those models, the system (11) is reduced to the second order dynamical system

$$
\frac{d x}{d \tau}=y, \frac{d y}{d \tau}=-b y\left[y^{2}+2 V(x)\right]^{1 / 2}-V^{\prime}(x) .
$$


For Einstein frame expanding models, the Hubble function is expressed in terms of the solution of (15) by (13) with $\epsilon=0$. The critical points for (15) are the points of the phase plane $(x, y)$ with the coordinates $\left(x_{c}, 0\right)$ where $V^{\prime}\left(x_{c}\right)=0, V\left(x_{c}\right) \geqslant 0$. For the corresponding solution the Hubble function is a constant, $H=H_{c}$, with $H_{c}^{2}=V_{E}\left(\phi_{c}\right) /\left[D(D-1) m_{P}^{D-1}\right]$. This solution describes the Minkowski spacetime for $V_{E}\left(\phi_{c}\right)=0$ and the de Sitter spacetime for $V_{E}\left(\phi_{c}\right)>0$. In the latter case for the cosmological constant one has $\Lambda=V_{E}\left(\phi_{c}\right) /\left(2 m_{P}^{D-1}\right)$.

The character of the critical points is determined by the eigenvalues

$$
\lambda_{1,2}=-b \sqrt{V_{c} / 2} \pm \sqrt{b^{2} V_{c} / 2-V_{c}^{\prime \prime}},
$$

where $V_{c}=V\left(x_{c}\right), V_{c}^{\prime \prime}=V^{\prime \prime}\left(x_{c}\right)$. For $V_{c}^{\prime \prime}<0\left(x_{c}\right.$ is a maximum of the potential $V(x))$ the critical point is a saddle. The directions of the corresponding separatrices are determined by the unit vectors $\mathbf{n}^{(i)}=\left(1, \lambda_{i}\right) / \sqrt{1+\lambda_{i}^{2}}$, $i=1,2$. For $V_{c}^{\prime \prime}>0\left(x_{c}\right.$ is a minimum of the potential $\left.V(x)\right)$ two cases should be considered separately. When $0<V_{c}^{\prime \prime}<b^{2} V_{c} / 2$, the critical point is a stable node. For $0<b^{2} V_{c} / 2<V_{c}^{\prime \prime}$ the critical point is a stable sink. In the case $V_{c}>0, V_{c}^{(i)} \equiv\left(d^{i} V / d x^{i}\right)_{x=x_{c}}=0$ for $i=1, \ldots, n-1$, and $V_{c}^{(n)} \neq 0$, the critical point is (i) a saddle for even $n$ and $V_{c}^{(n)}<0$, (ii) a stable node for even $n$ and $V_{c}^{(n)}>0$, (iii) a degenerate critical point with one stable node sector and with two saddle sectors for odd $n$. Another degenerate case corresponds to $V_{c}=0$ and $V_{c}^{\prime \prime}>0$. In this case the critical point is a stable sink.

If the value of the potential at the minimum is negative, then there is a classically forbidden region in the phase plane $(x, y)$. This region is determined by the inequality $y^{2}+2 V(x)<0$. At the boundary of the forbidden region, given by $y^{2}+2 V(x)=0$, one has $H=0$ and $d H / d t=$ $m_{P}^{1-D} V_{E}(\phi) /(D-1)<0$. Hence, at the boundary the expansion stops at a finite value of the cosmological time $t$ and then the model enters the stage of the contraction $(H<0)$. The corresponding dynamics is described by the dynamical system (15) with the opposite sign of the first term in the right-hand side of the second equation. For nonnegative potentials the expansion-contraction transition in models with flat space is not classically allowed.

We should also consider the behavior of the phase trajectories at the infinity of the phase plane. With this aim, it is convenient to introduce polar coordinates $(\rho, \theta)$ defined as $x=\rho \cos \theta /(1-\rho)$ and $y=\rho \sin \theta /(1-\rho)$, with $0 \leqslant \rho \leqslant 1,0 \leqslant \theta \leqslant 2 \pi$. Now the phase space is mapped onto a unite circle. The points at infinity correspond to $\rho=1$. For the potentials having the asymptotic behavior $V(x) \sim B|x|^{m}, m<4$, in the limit $x \rightarrow \infty$ one has the following critical points on the circle $\rho=1$. The points $\theta=0$ and $\theta=\pi$ are stable nodes for $m<0$ and saddles with two sectors for $m>0$. In the 
latter case the sectors are separated by a special solution described by the trajectory

$$
y(x) \approx-\frac{V^{\prime}(x)}{b \sqrt{2 V(x)}} \sim \frac{m}{b} \sqrt{B / 2}|x|^{m / 2-1},
$$

for $|x| \rightarrow \infty$. In the vicinity of the points $\theta=\pi / 2$ and $\theta=3 \pi / 2$ the potential terms can be neglected and these points are unstable degenerate nodes. For $m=4$ the nature of the critical points at $\theta=\pi / 2$ and $\theta=3 \pi / 2$ remains the same. In this case the other critical points correspond to $\theta=-\arctan (\sqrt{8 B} / b)$ and $\theta=\pi-\arctan (\sqrt{8 B} / b)$. The phase portrait near these points have two saddle sectors which are separated by the trajectory corresponding to the special solution (17). For $m>4$ there are two critical points on the circle $\rho=1$ corresponding to $\theta=\pi / 2$ and $\theta=3 \pi / 2$. These points are degenerate and have an unstable node sector and a saddle sector separated by the special solution (17). Similar behavior of the phase trajectories at the infinity takes place for the potentials with the asymptotic behavior $V(x) \sim B e^{\sigma|x|}, \sigma>0$, for $x \rightarrow \infty$ and for the values of the parameter $0<\sigma<2 b$. The separatrix between the saddle and node sectors is described by the special solution $y \approx-\operatorname{sgn}(x) \sigma \sqrt{2 B} e^{\sigma|x| / 2} / \sqrt{4 b^{2}-\sigma^{2}}$ for $x \rightarrow \infty$. The general solution behaves as $y \approx-\operatorname{sgn}(x) C e^{b|x|}$, with a positive constant $C$. This behavior coincides with that in the absence of the potential. For $\sigma \geqslant 2 b$ the dynamical system (15) has no critical points at infinity (on the circle $\rho=1$ ).

\section{Special cases}

A number of specific choices for the function $f(R)$ have been discussed in the literature. In the models with quantum corrections to the EinsteinHilbert Lagrangian the function $f(R)$ is of the polynomial form. A similar structure is obtained in the string-inspired models with the effective action expanded in powers of the string tension. However, it should be noted that in both these types of models coming from high-energy physics, the Lagrangian density in addition to the scalar curvature contains other scalars constructed from the Riemann tensor. In this context, the $f(R)$ theories can be considered as models simple enough to be easy to handle from which we gain some insight in modifications of gravity. In some models proposed for dark energy the function $f(R)$ contains terms with the inverse power of the Ricci scalar. For one of the first models of this type $f(R)=m_{P}^{D-1}(-R+$ $\gamma / R^{m}$ ) with $\gamma$ and $m>0$ being constants (Capozziell 2002). However, there is a matter instability problem in these models. The model with an additional term $\beta R^{2}$ in the brackets has been discussed in Brookfield et al. (2006). Models containing in $f(R)$ exponential functions of the form $e^{\gamma R}$ and providing the accelerating cosmological solutions without a future singularity are considered in Cognola et al. (2008). Examples of the $f(R)$ 
functions, containing combinations of the powers and exponentials of $R$, that allow to construct models with a late-time accelerated expansion consistent with local gravity constraints, are studied in references Hu \& Sawicki (2007) (see also Nojiri \& Odintsov (2011) and Clifton et al. (2012)). For example, in the Tsujikawa model $f(R)=m_{P}^{D-1}\left[-R+\gamma \tanh \left(R / R_{0}\right)\right]$, whereas in the $\mathrm{Hu} \%$ Sawicki model $f(R)=m_{P}^{D-1}\left[-R+\gamma\left(1+\left(R / R_{0}\right)^{-m}\right)\right]$ with constants $\gamma$ and $R_{0}$.

As an application of general analysis given above, first let us consider a $(D+1)$-dimensional generalization of the Starobinsky model (see [?] for the discussion of inflation in this type of models). The corresponding lagrangian density is taken as

$$
f(R)=m_{P}^{D-1}\left(-R+\beta R^{2}\right),
$$

where $\beta$ is a constant. The potential in terms of the canonical scalar field is written in the form

$$
V(x)=V_{0} \exp \left(\frac{D-3}{D-1} x\right)\left(1-e^{-x}\right)^{2},
$$

where $V_{0}=\left(t_{0} / \phi_{0}\right)^{2} m_{P}^{D-1} /(4 \beta)$. For $\beta>0$ and $x \neq 0$ the potential (19) is positive. It has a minimum at $x=0$ with $V(0)=0$. In figure 1 we have plotted the potential (19) as a function of $\phi / \phi_{0}$ for $D=3,4,5$ (numbers near the curves). As is seen from the graphs, in the case $D=3$ an inflationary plateau appears for large values of $\phi / \phi_{0}$ which corresponds to the Starobinsky inflation. Hence, from the point of view of the Starobinsky inflation, the spatial dimension $D=3$ is special.

In the limit $x \rightarrow-\infty$ the potential behaves as $\exp [-(D+1) x /(D-1)]$. From here it follows that the point $\rho=1, \theta=\pi / 2$ is degenerate having an unstable node sector and a saddle sector (see figure 2). In the limit $x \rightarrow+\infty$ one has $V(x) \propto \exp [(D-3) x /(D-1)]$ and for $D>3$ the behavior of the phase trajectories near the point $\rho=1, \theta=3 \pi / 2$ is similar to that for the point $\rho=1, \theta=\pi / 2$. In the special case $D=3$ the dynamical system has a critical point at $\rho=1, \theta=0$. This point is a node (see the left panel in figure 2) and the corresponding unstable separatarix describes an inflationary expansion. This special solution is an attractor for the general solution. For $D=3$ the point $\rho=1, \theta=3 \pi / 2$ at the infinity of the phase plane is an unstable node. The only critical point in the finite region of the phase plane, $(x, y)=(0,0)$, corresponds to the minimum of the potential. This point is a stable sink and the corresponding geometry is the Minkowski spacetime. The phase portrait, mapped on the unit circle, is presented in the left panel of figure 2 for $D=3$ and in the right panel for $D>3$.

For the model

$$
f(R)=m_{P}^{D-1}\left(-R+\beta_{n} R^{n}\right),
$$

with even $n$ and $\beta_{n}>0$, the potential is nonnegative and is given by the 

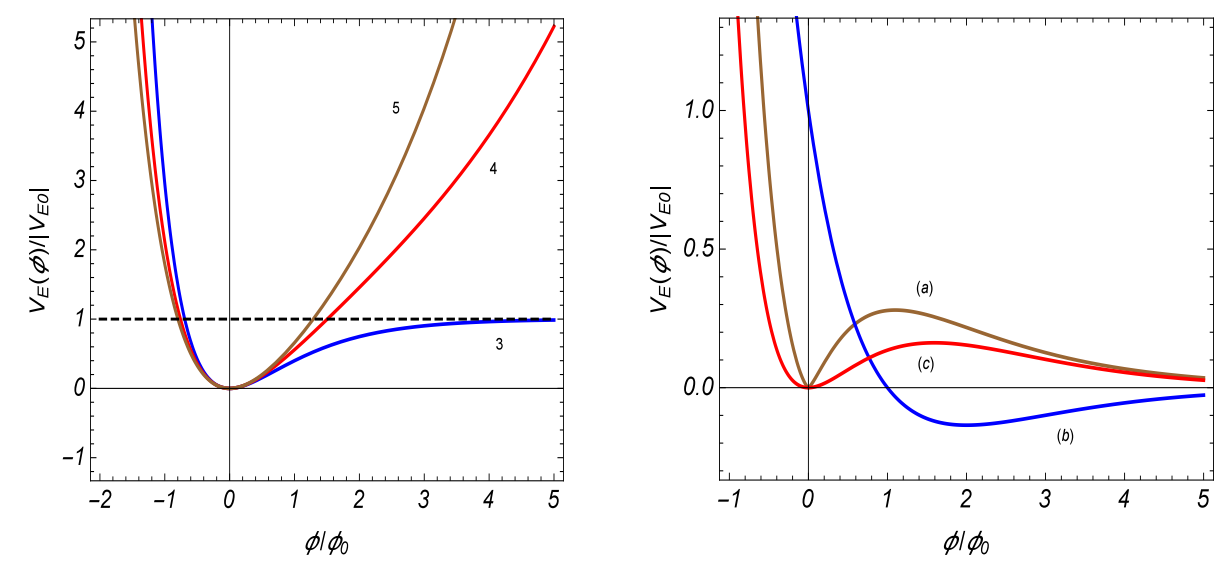

Figure 1: The left panel presents the Einstein frame potentials in the Starobinsky model for different values of the spatial dimension (numbers near the curves). On the right panel the potentials corresponding to the $f(R)$ functions (20) (for $n=4$, curve (a)), (22) (curve (b)) and (25) (curve (c)) are plotted.
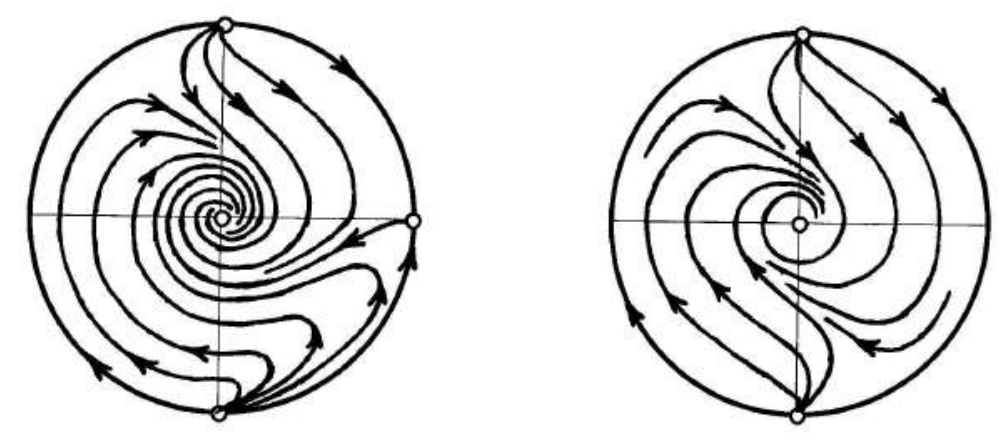

Figure 2: Phase portraits of the dynamical system for a $(D+1)$-dimensional generalization of the Starobinsky model in the cases $D=3$ (left panel) and $D>3$ (right panel). 
expression:

$$
V(\phi)=V_{0} \exp \left(-\frac{D+1}{D-1} x\right)\left[\left(1-e^{x}\right)^{n}\right]^{1 /(n-1)},
$$

where $V_{0}=(n-1)\left(t_{0} / \phi_{0}\right)^{2} n^{n /(1-n)} \beta_{n}^{1 /(1-n)} m_{P}^{D-1}$. For $n=2$ the potential is reduced to the one for the Starobinsky model. In the limit $x \gg 1$ one has $V(x) \rightarrow+\infty$ for $n<(D+1) / 2$ and $V(x) \rightarrow 0$ for $n>(D+1) / 2$. For $n=(D+1) / 2$, in the limit $x \rightarrow+\infty$ the potential has a nonzero plateau: $V(\phi) \rightarrow V_{0}$. The potential (21) for $n=4$ and $D=3$ is depicted in the right panel of figure 1 (graph (a)). For $n>(D+1) / 2$ (see graph (a) in the right panel of figure 1) we have two critical points in the finite region of the phase plane. The first one, $(x, y)=(0,0)$, corresponds to the minimum of the potential and is a stable sink. The second one, $(x, y)=\left(x_{c}, 0\right)$, corresponds to the maximum of the potential and is a saddle. The phase portrait is depicted in the left panel of figure 3. At infinity of the phase plane, the nature of the point $\rho=1, \theta=\pi / 2$ remains the same as in the previous example, whereas the point $\rho=1, \theta=3 \pi / 2$ becomes an unstable node. In the region $x \gg 1$ and for $n>(D+1) / 2$ the special solution is an attractor for a general solution in the limit $t \rightarrow+\infty$.

For the next example we take the function

$$
f(R)=f_{0} e^{\gamma R} .
$$

The corresponding potential takes the form

$$
V(x)=V_{0} \exp \left(-\frac{2}{D-1} x\right)\left[x+\ln \left(-M_{D+1}^{D-1} / \gamma f_{0}\right)-1\right],
$$

with $V_{0}=-\left(t_{0} / \phi_{0}\right)^{2} m_{P}^{D-1} / \gamma$. For $|\gamma R| \ll 1$ one has $f(R)=f_{0}+f_{0} \gamma R$. Taking $f_{0} \gamma=-m_{P}^{D-1}$, the linear in $R$ term coincides with the HilbertEinstein lagrangian density. With this choice the potential simplifies to

$$
V(x)=V_{0} \exp \left(-\frac{2 x}{D-1}\right)(x-1) .
$$

The graph of this potential for $\gamma>0$ is plotted in the right panel of figure 1 (curve (b)). The value of the potential at the minimum is negative. Note that in this case for $|\gamma R| \ll 1$ the model reduces to General Relativity with a negative cosmological constant.

In the case of the function

$$
f(R)=f_{0}\left(e^{\gamma R}-1\right),
$$

with $f_{0} \gamma=-m_{P}^{D-1}$ and for small curvatures, corresponding to $|\gamma R| \ll 1$, the model is reduced to General Relativity with zero cosmological constant. The corresponding potential is given by the expression

$$
V(x)=V_{0} \exp \left(-\frac{D+1}{D-1} x\right)\left[1+e^{x}(x-1)\right],
$$


with the same notation $V_{0}$ as in (24). This potential for $\gamma<0\left(V_{0}>0\right)$ is plotted in figure 1 (curve (c)).

In the case of the function (22) with $f_{0} \gamma=-m_{P}^{D-1}$ and $\gamma>0$ a characteristic feature of the potential is the presence of the region in the field space where it is negative. For this type of potentials there is a classically forbidden region determined by $y^{2}+2 V(x)<0$. As it has been noted above, at the boundary of this region the expansion stops at a finite value of the cosmological time $t$ and then the model enters the stage of the contraction. For the potential (24), the only critical points of the dynamical system (15) are at the infinity of the phase plane. The corresponding phase portrait is depicted in the right panel of figure 3. The classically forbidden region of the phase space is shaded. The full/dashed trajectories correspond to the expansion/contraction phases. As it follows from (15), the trajectories for the contraction stage are obtained from those describing an expansion by the transformation $\tau \rightarrow-\tau, y \rightarrow-y$. For expanding models, near the point $\rho=1, \theta=\pi / 2$ the phase portrait has two sectors: an unstable node sector and a saddle sector. The point $\rho=1, \theta=3 \pi / 2$ is an unstable node. Depending on the initial conditions, the expanding models start their evolution at finite cosmic time $t=t_{i}$ from the point $\rho=1, \theta=\pi / 2$ or from the point $\rho=1, \theta=3 \pi / 2$. During a finite time interval the trajectories reach the boundary of the forbidden region at $t=t_{c}>t_{i}$. At this moment the expansion stops $\left(H\left(t_{c}\right)=0\right)$ and the model enters the contraction stage (dashed trajectories on the phase portrait). The corresponding trajectories enter the critical points $\rho=1, \theta=3 \pi / 2$ and $\rho=1, \theta=\pi / 2$ at finite time $t_{f}>t_{c}$. Hence, all the models have a finite lifetime $t_{f}-t_{i}$.

For the function (25) with $f_{0} \gamma=-m_{P}^{D-1}$ the potential is given by the expression (26). In the case $\gamma<0$ the qualitative behavior of this potential is similar to that for the function (20) with $n>(D+1) / 2$ and the corresponding phase portrait is qualitatively equivalent to the one presented in the left panel of figure 3. However, note that the asymptotic behavior of the potential in the limit $\phi \rightarrow+\infty$ is not purely exponential. For the corresponding potential in $(15)$ from $(26)$ one has $V(x) \approx V_{2} x e^{-2 x /(D-1)}$ in the limit $x \gg 1$. Here, $V_{2}$ is expressed in terms of the coefficient $V_{0}$ in (26). It can be seen that the dynamical system has a special solution with the asymptotic behavior $y^{2} \approx 2 V(x) /\left(D^{2}-1\right)$ in the region $x \gg 1$. This special solution is an attractor for the general solution near the critical point $(\rho, \theta)=(1,0)$. The corresponding time dependence of the scalar field is determined from the relation $2 V(x) \approx(D-1)^{2}\left(D^{2}-1\right) / \tau^{2}$, which is obtained by the integration of the first equation in (15). With the help of this relation, the asymptotic behavior of the Einstein frame scale factor is found: $a(t) \approx a_{0}\left(t / t_{0}\right)^{D}, t \rightarrow+\infty$. The asymptotic behavior of $x$ near the critical point $(\rho, \theta)=(1,0)$, as a function of the time coordinate is simpler in the Jordan frame. By using the expression for the function $y(x)$ and the relation $d t_{J}=e^{-x /(D-1)} d t$, in the region $x \gg 1$ we can see that 
$x \approx V_{2} \tau_{J}^{2} /\left[2\left(D^{2}-1\right)\right]$, where $\tau_{J}=t_{J} / t_{0}$. For the scale factor in the Jordan frame one gets $a_{J}\left(t_{J}\right) \approx$ const $e^{x}$.
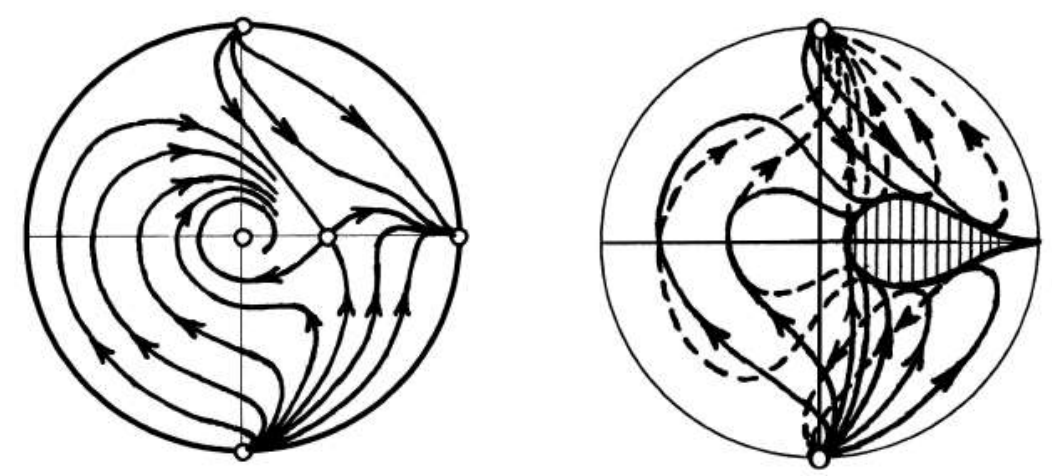

Figure 3: Phase portraits of the dynamical system for the potential (21), with $n=4$, and for the potential (24).

\section{Conclusion}

We have considered the qualitative evolution of cosmological models in $(D+1)$-dimensional $f(R)$ gravity. In order to do that the model is transformed to an equivalent model described by a scalar-tensor theory. From the point of view of the description of the cosmological dynamics, the most convenient representation corresponds to the Einstein frame, in which the gravitational part of the action coincides with that for General Relativity. In this frame there is a direct interaction of the scalar field with a nongravitational matter.

For homogeneous and isotropic cosmological models with flat space the equations can be presented in the form of a third order autonomous dynamical system (11). The corresponding phase space has an invariant subspace describing the gravi-scalar models in the absence of a non-gravitational matter. For a general case of the function $f(R)$, we have found the critical points of the system and their nature, including the points at the infinity of the phase plane. As applications of general analysis, various special cases of the function $f(R)$ are considered.

\section{Acknowledgments}

R. M. A. and G. H. H. were supported by the Committee of Science Ministry of Education and Science RA, within the frame of the Project No. 18T-1C355. 


\section{References}

Birrell, N.D.; Davies, P.C.W. Quantum Fields in Curved Space, Cambridge University Press, Cambridge, 1982

Brookfield, A. W.; van de Bruck, C.; Hall, L.M.H. 2006, Phys. Rev. D, 74, 064028

Buchbinder, I.L., Odintsov, S.D., Shapiro, I.L. Effective Action in Quantum Gravity, IOP, Bristol, 1992

Capozziello, S. 2002, Int. J. Mod. Phys. D, 11, 483

Capozziello, S.; De Laurentis, M., 2011, Phys. Rept., 509, 167

Capozziello, S.; Francaviglia, M., 2008, Gen. Rel. Grav., 357

Clifton, T.; Ferreira, P.G.; Padilla, A.; Skordis, . 2012, Phys. Rept., 513,1

Cognola, G.; Elizalde, E.; Nojiri, S.; Odintsov, S.D., Sebastiani, L.; Zerbini, S. 2008, Phys. Rev. D, 77, 046009

De Felice, A.; Tsujikawa, S. 2010, Living Rev. Rel., 13, 3

Hu, W.; Sawicki, I. 2007, Phys. Rev. D, 76, 064004

Maeda, K. 1988, Phys. Rev. D, 37, 858

Nojiri, S.; Odintsov, S. D. 2007, Int. J. Geom. Meth. Mod. Phys., 4, 115

Nojiri, S.; Odintsov, S. D. 2011, Phys. Rept. 59

Saharian, A.A. 1999, Class. Quantum Grav., 16, 2057

Saharian, A.A. 2000a, Astrophysics, 43, 92

Sotiriou, T.P.; Faraoni, V. 2010, Rev. Mod. Phys., 451

Starobinsky, A.A. 1980, Phys. Lett. B, 91, 99

Will C.M., Theory and Experiment in Gravitational Physics, Cambridge University Press, Cambridge, 2018 